\title{
Brief 46: Aktennotiz für den Landesschulrat Buhl (17.10.1956)
}

Aktennotiz für Landesschulrat Buhl ${ }^{1}$

17.10.1956.

1. Eine wesentliche Aufgabe der Lehrtätigkeit in der hochschulmässigen Lehrerbildung ist die Erziehung der künftigen Lehrer zu selbständigem Fragen und Arbeiten. Anregungen und Impulse dafür werden für den Dozenten der Pädagogischen Hochschule stark von der eigenen wissenschaftlichen Arbeit ausgehen. Darum ist es erforderlich, dass der Lehrerbildner im Kontakt mit der Forschung seiner Wissenschaft bleibt. Wenn es für eine besondere Aufgabe zweckmässig erscheint, sollten Professoren und Dozenten der Pädagogischen Hochschulen für eine begrenzte Zeit beurlaubt werden, um an Universitäten oder wissenschaftlichen Instituten in ihrem Fach weitere Anregungen zu erhalten.

2. Eine besondere Aufgabe liegt hier im Fachbereich Physik-Philosophie vor. Die Entwicklung der modernen Physik wird von der Öffentlichkeit mit grossem Interesse verfolgt. Dieses Interesse richtet sich nicht nur auf die weitreichenden technischen Möglichkeiten, die die Atomphysik erschlossen hat, sondern ebenso sehr auch auf die Wandlungen im Verständnis der Naturvorgänge, die sich mit dem Übergang von der klassischen zur modernen Physik angebahnt haben. Nun ist aber für den Laien die moderne Physik, trotz aller popularisierenden Einführungen, im Ganzen ein Buch mit sieben Siegeln. Das Interesse an ihr verführt zu unkritischem Mitreden und Mitdeuten von Vorgängen, deren Verständnis erst in sachlicher und kritischer Zusammenarbeit von Physik und Philosophie gewonnen werden muss. Von der modernen Physik her sind Argumente für die verschiedensten weltanschaulichen Ideologien herangeholt worden. Darin dürfte eine Gefährdung der Bildung in unserem Volk liegen, der wir gerade in der Lehrerbildung entgegentreten sollten. Die naturphilosophische Arbeit, für die sich Frau Henry Zeit und Musse erbittet, steht insofern auch inhaltlich in direktem Zusammenhang mit ihrem philosophisch-pädagogischem Lehrauftrag an der Pädagogischen Hochschule.

1 Handschriftlich hinzugefügt

(C) Springer Fachmedien Wiesbaden GmbH, ein Teil von Springer Nature 2019

K. Herrmann (Hrsg.), Grete Henry-Hermann: Philosophie - Mathematik-

Quantenmechanik, Frauen in Philosophie und Wissenschaft. Women

Philosophers and Scientists, https://doi.org/10.1007/978-3-658-16241-2_68 